\title{
Finding the Unique Permutation Matrix for Reverse Order Kronecker Product Intuitively
}

\author{
Muhammad G. Ali, Shoab A. Khan, and Sajid G. Khawaja
}

\begin{abstract}
With the adverse research in field of engineering and technology, the use of Kronecker product highlighted from many aspects by researchers in different fields of science, especially considering digital signal processing where Discrete Fourier Transform plays a supreme role. This transform can be performed using Kronecker product in an iterative way. The implementation of DFT using this product needs a reverse order multiplication that can easily be catered for by applying few techniques of linear algebra i.e. by using a unique permutation matrix, on the original Kronecker Product. The permutation matrix obtained from "I" interchanging of rows / columns from an identity matrix. A total of $n$ ! permutation matrices will be obtained using this methodology. This paper presents a technique for finding the unique permutation matrix out of $n$ ! matrices. This uniquely identified permutation matrix is used to attain the reverse order Kronecker product without using the same technique, as used for obtaining the original Kronecker product.
\end{abstract}

Index Terms-Discrete fourier transform (DFT), digital signal processing (DSP), intuitive method, kronecker product (KP), permutation matrix (PM).

\section{INTRODUCTION}

Kronecker product, plays an imperative role in major disciplines of science as in mathematics, linear algebra, big data analysis and signal processing etc. that acts as a nucleus in the formation of this modern era especially from application perspective [1]. This product also finds its applications in matrix calculus [2], system theory [3], differential equations [4] that are the basis for circuit analysis and much more. A well-established transform to study a time domain signal from frequency perspective, filtering and analysis is Fourier transform [5]. Kronecker product is an arithmetic tool that finds its significance in many applications in field of research. Usage of this product for the modular design of computational structures for signal processing algorithms especially on FPGA [6] can also be implemented. The given product finds its applications in usage of Krylov Subspace methods for the evaluation of the expressions as $f(A) b$ using Kronecker structure on $A$ [7], execution, implementation of applications of Hadamard matrices [8], recursive generations of a large class of discrete unitary transforms [9], Image rescaling, reconstruction [10], matrix calculus [11] and so on. Its usage on FPGA [6], Graphical processing Units [12] etc. for parallel computation of signal

Manuscript received August 19, 2019; revised October 10, 2019

Muhammad G. Ali, Shoab A. Khan, and Sajid G. Khawaja are with Department of Computer Engineering, College of Electrical and Mechanical Engineering, National University of Sciences and Technology, Pakistan (e-mail: mgali17@ce.ceme.edu.pk, shoabakhan@ceme.nust.edu.pk, sajid.gul@ceme.nust.edu.pk). processing algorithms cannot be rendered.

The main problem that arises during the implementation of the Kronecker product is its computation cost, especially to those algorithms in which it is used repeatedly. This problem becomes a bottleneck while computing two or more products simultaneously, especially when, in the same arithmetic calculation original and reverse order Kronecker product are to be calculated, e.g. in formulation of DFT as stated in (1), that is taken as a building block in this paper. We can improve the performance of various algorithms using this product by optimizing its performance with respect to computational time.

Keeping in view the calculation of Kronecker product, an intuitive approach is proposed to bypass the rigorous calculations required to compute the permutation matrix. In this paper, we present a mechanism that calculates a unique permutation matrix, so that computation time for finding the reverse order product decreases, and reverse order Kronecker product from its original may be generated with minimum computations.

The paper is structured in the following manner as Section II discusses the background and working of permutation matrix, proposed methodology is discussed in Section III which is in the continuation with the observations and results in Section IV, leading us to conclusion in Section V that meets up with the end of the paper.

\section{BACKGROUND}

In this section, we highlight the basic mathematics needed to calculate the required uniquely identified permutation matrix for Kronecker product to calculate its reverse order Product.

The usage of Kronecker and reverse order KP in same arithmetic calculation finds its application in formulation of DFT, in which $F_{n}$ is an $n \times n$ DFT matrix, $I_{n}$ being $n \times n$ identity matrix and $D_{n^{2}}$ is a diagonal matrix leading us to a bit reversed order $n^{2}$ DFT matrix as $F_{n^{2}}^{\prime}$.

$$
F_{n^{2}}^{\prime}=\left(I_{n} \otimes F_{n}\right) D_{n^{2}}\left(F_{n} \otimes I_{n}\right)
$$

In such cases where we have to compute the original and reverse order KP at same time, being it to be computationally expensive algorithm, we can use Permutation Matrix for this purpose to reuse the previously calculated product for further use as below:

$$
F_{n^{2}}^{\prime}=\left(I_{n} \otimes F_{n}\right) D_{n^{2}} P_{n^{2}}\left(F_{n} \otimes I_{n}\right) P_{n^{2}}
$$

And hence by using the permutation matrix, we can find the DFT with less computation using the previously calculated Kronecker Product. 


\section{A. Permutation Matrix}

Permutation matrix is a binary matrix having two entries (i.e. 0 \& 1), and is obtained by permuting the columns /rows of an $n x n$ identity matrix. Note that every permutation in the identity matrix provides us with a unique permutation matrix that leads us towards different solutions after its multiplication with original matrix. For instance, let us suppose a $4 \times 4$ matrix, multiplied with different permutation matrices of same order as below:

$\left[\begin{array}{llll}a_{11} & a_{12} & a_{13} & a_{14} \\ a_{21} & a_{22} & a_{23} & a_{24} \\ a_{31} & a_{32} & a_{33} & a_{34} \\ a_{41} & a_{42} & a_{43} & a_{44}\end{array}\right]\left[\begin{array}{llll}1 & 0 & 0 & 0 \\ 0 & 0 & 0 & 1 \\ 0 & 1 & 0 & 0 \\ 0 & 0 & 1 & 0\end{array}\right]=\left[\begin{array}{llll}a_{11} & a_{13} & a_{14} & a_{12} \\ a_{21} & a_{23} & a_{24} & a_{22} \\ a_{31} & a_{33} & a_{34} & a_{32} \\ a_{41} & a_{43} & a_{44} & a_{42}\end{array}\right]$

Using a different permutation matrix of same order as:

$\left[\begin{array}{llll}a_{11} & a_{12} & a_{13} & a_{14} \\ a_{21} & a_{22} & a_{23} & a_{24} \\ a_{31} & a_{32} & a_{33} & a_{34} \\ a_{41} & a_{42} & a_{43} & a_{44}\end{array}\right]\left[\begin{array}{llll}0 & 0 & 0 & 1 \\ 0 & 0 & 1 & 0 \\ 1 & 0 & 0 & 0 \\ 0 & 1 & 0 & 0\end{array}\right]=\left[\begin{array}{llll}a_{13} & a_{14} & a_{12} & a_{11} \\ a_{23} & a_{24} & a_{22} & a_{21} \\ a_{33} & a_{34} & a_{32} & a_{31} \\ a_{43} & a_{44} & a_{42} & a_{41}\end{array}\right]$

Hence from (3) \& (4), using different permutation matrices multiplied with same matrix, different results are obtained.

\section{B. Rows and Columns Permuted Matrices}

From (3) \& (4), it is observed that in matrix multiplication, using PM after the original matrix swaps the columns of the original matrix. However, to achieve a row permuted matrix, the permutation matrix is placed behind the original matrix as below:

$$
\left[\begin{array}{llll}
0 & 0 & 0 & 1 \\
0 & 0 & 1 & 0 \\
1 & 0 & 0 & 0 \\
0 & 1 & 0 & 0
\end{array}\right]\left[\begin{array}{llll}
a_{11} & a_{12} & a_{13} & a_{14} \\
a_{21} & a_{22} & a_{23} & a_{24} \\
a_{31} & a_{32} & a_{33} & a_{34} \\
a_{41} & a_{42} & a_{43} & a_{44}
\end{array}\right]=\left[\begin{array}{llll}
a_{41} & a_{42} & a_{43} & a_{44} \\
a_{31} & a_{32} & a_{33} & a_{34} \\
a_{11} & a_{12} & a_{13} & a_{14} \\
a_{21} & a_{22} & a_{23} & a_{24}
\end{array}\right]
$$

So from (4) and (5) it can be observed that for same $n \times n$ permutation matrix, the placement of the PM will decide the resultant matrix either to be a row permuted matrix or a column permuted matrix.

\section{Kronecker Product and Permutation Matrix}

Let us suppose we have $A$ and $B$ matrices of $m x n$ and $p x q$ order respectively, then the Kronecker product of $A \& B$ would be as

$$
A \otimes B=\left[\begin{array}{ccc}
a_{11} * \mathbf{B} & \ldots & a_{1 n} * \mathbf{B} \\
\vdots & \ddots & \vdots \\
a_{m 1} * \mathbf{B} & \cdots & a_{m n} * \mathbf{B}
\end{array}\right]
$$

That can be expressed more explicitly as

$$
\left[\begin{array}{ccccccccc}
a_{11} b_{11} & a_{11} b_{12} & \cdots & a_{11} b_{1 q} & \cdots & a_{1 n} b_{11} & \cdots & \cdots & a_{1 n} b_{1 q} \\
a_{11} b_{21} & a_{11} b_{22} & \cdots & a_{11} b_{2 q} & \cdots & a_{1 n} b_{21} & \cdots & \cdots & a_{1 n} b_{2 q} \\
\vdots & \vdots & \ddots & \vdots & \cdots & \vdots & \cdots & \ddots & \vdots \\
a_{11} b_{p 1} & a_{11} b_{p 2} & \cdots & a_{11} b_{p q} & \cdots & a_{1 n} b_{p 1} & \cdots & \cdots & a_{1 n} b_{p q} \\
\vdots & \vdots & \ddots & \vdots & \ddots & \vdots & \vdots & \ddots & \vdots \\
\vdots & \vdots & \ddots & \vdots & \ddots & \vdots & \vdots & \ddots & \vdots \\
a_{m 1} b_{11} & a_{m 1} b_{12} & \cdots & a_{m 1} b_{1 q} & \cdots & a_{m n} b_{11} & \cdots & \cdots & a_{m n} b_{1 q} \\
a_{m 1} b_{21} & a_{m 1} b_{22} & \cdots & a_{m 1} b_{2 q} & \cdots & a_{m n} b_{21} & \cdots & \cdots & a_{m n} b_{2 q} \\
\vdots & \vdots & \ddots & \vdots & \ddots & \vdots & \vdots & \ddots & \vdots \\
a_{m 1} b_{p 1} & a_{m 1} b_{p 2} & \cdots & a_{m 1} b_{p q} & \cdots & a_{m n} b_{p 1} & \cdots & \cdots & a_{m n} b_{p q}
\end{array}\right]
$$

And the reverse product i.e. $B \otimes A$ can be written as

$$
B \otimes A=\left[\begin{array}{ccc}
b 11 * \mathbf{A} & \ldots & b 1 p * \mathbf{A} \\
\vdots & \ddots & \vdots \\
b p 1 * \mathbf{A} & \cdots & b p q * \mathbf{A}
\end{array}\right]
$$

From (6) and (7) it can be observed that $A \otimes B$ and $B \otimes A$ are entirely different matrices. But from $A \otimes B, B \otimes A$ can be achieved just by swapping some of the rows and columns with the specific permutations. In other words we can say that $A \otimes B$ and $B \otimes A$ are permutation equivalent matrices, and there exists a unique permutation matrix such that

$$
B \otimes A=P(A \otimes B) P^{T}
$$

Furthermore, another characteristic of this unique permutation matrix is that the transpose and inverse of the unique PM is same as the PM itself, as

$$
P=P^{-1}=P^{T}
$$

All remaining $n x n$ PM's, doesn't qualify the above property due to which they are not classified as the unique PM for Kronecker Product

\section{Proposed Methodology}

In this section, we propose a methodology for calculation of reverse order Kronecker using unique permutation matrix. For this, let us suppose two, $2 \times 2$ matrices $A$ and $B$ for understanding. The two Kronecker products $A \otimes B$, as in (6), and $B \otimes A$, as in (7) will be as follows,

$$
A \otimes B=\left[\begin{array}{llll}
a_{11} b_{11} & a_{11} b_{12} & a_{12} b_{11} & a_{12} b_{12} \\
a_{11} b_{21} & a_{11} b_{22} & a_{12} b_{21} & a_{12} b_{22} \\
a_{21} b_{11} & a_{21} b_{12} & a_{22} b_{11} & a_{22} b_{12} \\
a_{21} b_{21} & a_{21} b_{22} & a_{22} b_{21} & a_{22} b_{22}
\end{array}\right]
$$

Similarly

$$
B \otimes A=\left[\begin{array}{llll}
a_{11} b_{11} & a_{12} b_{11} & a_{11} b_{12} & a_{12} b_{12} \\
a_{21} b_{11} & a_{22} b_{11} & a_{21} b_{12} & a_{22} b_{12} \\
a_{11} b_{21} & a_{12} b_{21} & a_{11} b_{22} & a_{12} b_{22} \\
a_{21} b_{21} & a_{22} b_{21} & a_{21} b_{22} & a_{22} b_{22}
\end{array}\right]
$$

Total number of permutation matrices that can be produced from an $n^{\text {th }}$ order identity matrix are calculated as

$$
\text { no of PMs for } I_{n x n}=n \text { ! }
$$

As $A \& B$ are both $2 \times 2$ order matrices and the order of their Kronecker Product will be $4 \times 4$, thus in this case a $4 \times 4$ identity matrix will be used as PM that generates 4 ! i.e. 24 different permutation matrices as stated in (12) which are shown in Fig. 1.

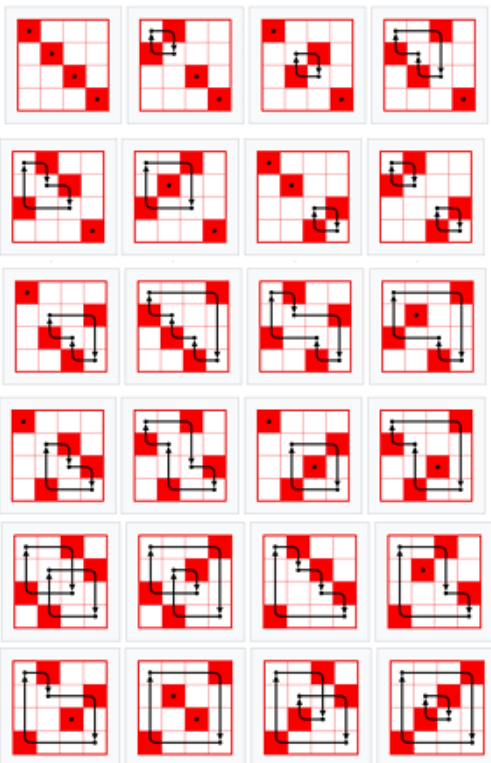

Fig. 1. Generation of 4 ! permutations of $4 \times 4$ identity. 
Out of these 24 matrices as shown in Fig. 1, a single unique permutation matrix say $P_{4}$ (eq.13) satisfies the requirements for reverse order Kronecker product as stated in (9). From the above two matrices (10) and (11) it can be observed that from $A \otimes B$ we can easily produce $B \otimes A$ just by swapping second and third rows and then swapping same columns. To achieve this, an identity matrix will be used, that is also swapped in the same manner, and is then multiplied as in (4) $\&(5)$.

$$
P_{4}=\left[\begin{array}{llll}
1 & 0 & 0 & 0 \\
0 & 0 & 1 & 0 \\
0 & 1 & 0 & 0 \\
0 & 0 & 0 & 1
\end{array}\right]
$$

That is same number of rows are swapped from the identity matrix to make unique permutation matrix, that satisfies the unique PM property and hence by substituting, it may become

$$
\begin{aligned}
& P M * B \otimes A * P M \\
& =\left[\begin{array}{llll}
1 & 0 & 0 & 0 \\
0 & 0 & 1 & 0 \\
0 & 1 & 0 & 0 \\
0 & 0 & 0 & 1
\end{array}\right]\left[\begin{array}{llll}
a_{11} b_{11} & a_{12} b_{11} & a_{11} b_{12} & a_{12} b_{12} \\
a_{21} b_{11} & a_{22} b_{11} & a_{21} b_{12} & a_{22} b_{12} \\
a_{11} b_{21} & a_{12} b_{21} & a_{11} b_{22} & a_{12} b_{22} \\
a_{21} b_{21} & a_{22} b_{21} & a_{21} b_{22} & a_{22} b_{22}
\end{array}\right]\left[\begin{array}{llll}
1 & 0 & 0 & 0 \\
0 & 0 & 1 & 0 \\
0 & 1 & 0 & 0 \\
0 & 0 & 0 & 1
\end{array}\right] \\
& =A \otimes B
\end{aligned}
$$

These operations will eventually give us the results, that corresponds to our reverse order Kronecker Product i.e. $A \otimes B$.

\section{A. Formation of $n \times n$ Permutation Matrix}

In order to find the uniquely identified permutation matrix that satisfies requirements for reverse order Kronecker calculation stated in (6) and (7), we take two matrices $A$ and $B$ of order $2 \times 2$ having Kronecker product of order $4 \times 4$ as discussed earlier

$$
A \otimes B \Rightarrow \begin{array}{llll}
\left(1^{s t}\right) & \left(2^{n d}\right) & \left(3^{r d}\right) & \left(4^{\text {th }}\right) \\
a_{11} b_{11} & a_{11} b_{12} & a_{12} b_{11} & a_{12} b_{12} \\
a_{11} b_{21} & a_{11} b_{22} & a_{12} b_{21} & a_{12} b_{22} \\
a_{21} b_{11} & a_{21} b_{21} & a_{22} b_{11} & a_{22} b_{12} \\
a_{21} b_{12} & a_{22} b_{22} & a_{22} b_{21} & a_{22} b_{22}
\end{array}
$$

Whereas it's reverse order Kronecker product would be as

$$
\mathrm{B} \otimes \mathrm{A} \Rightarrow \begin{array}{cccc}
\left(1^{s t}\right) & \left(2^{\text {nd }}\right) & \left(3^{r d}\right) & \left(4^{\text {th }}\right) \\
a_{11} b_{11} & a_{12} b_{11} & a_{11} b_{12} & a_{12} b_{12} \\
a_{21} b_{11} & a_{22} b_{11} & a_{21} b_{12} & a_{22} b_{12} \\
a_{11} b_{21} & a_{12} b_{21} & a_{11} b_{22} & a_{12} b_{22} \\
a_{21} b_{21} & a_{22} b_{21} & a_{21} b_{22} & a_{22} b_{22}
\end{array}
$$

The swapping hierarchy of entries from $A \otimes B$ towards $B \otimes A$ is shown below. Furthermore, these are the swapped rows similar to the swapping of rows/columns of an identity matrix to form a uniquely identified PM,

$$
\begin{aligned}
{\left[\begin{array}{cccc}
1 & 2 & 3 & 4 \\
5 & 6 & 7 & 8 \\
9 & 10 & 11 & 12 \\
13 & 14 & 15 & 16
\end{array}\right] } & \longleftrightarrow\left[\begin{array}{cccc}
1 & 3 & 2 & 4 \\
9 & 11 & 10 & 12 \\
5 & 7 & 6 & 8 \\
13 & 15 & 14 & 16
\end{array}\right] \\
A \otimes B & \Leftrightarrow B \otimes A
\end{aligned}
$$

It can be observed in the above case that reverse order KP i.e. $B \otimes A$ can be attained from $A \otimes B$ by swapping the 2 nd and 3rd rows and then same columns or vice versa. Taking $A \otimes B$ and reshaping to make it $B \otimes A$ using the assigned labels would lead towards the formation of permutation matrix as below

$$
\begin{aligned}
& A \otimes B \rightarrow \underline{\underline{1}} 23 \quad 3 \quad \underline{\underline{4}} \\
& B \otimes A \rightarrow \underline{\underline{1}} 32 \underline{\underline{4}}
\end{aligned}
$$

It is observed that the 1 st and 4 th rows of both are at the same place (underlined) where as other 2nd and 3rd rows/columns are swapped with each other. Considering the stated hierarchy, from $A \otimes B$ the permutations of rows of $B \otimes A$ can be achieved as follows:

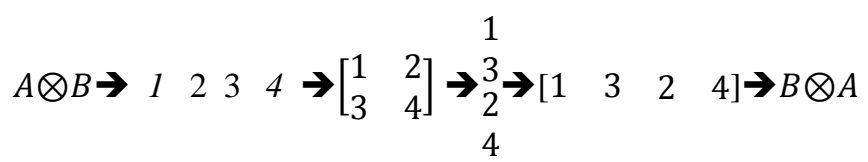

In order to transform $B \otimes A$ from $A \otimes B$, the matrix entries are first row permuted then column permuted. As stated earlier, the swapping hierarchy of the Kronecker product, to achieve its reverse order, is same as that of identity matrix. For the given example, the uniquely identified PM can be achieved using the same transitions of rows/columns as stated above. Here entries depict the placement of ' 1 ' in each row/column of identity matrix. This swapping technique would lead us towards the unique permutation matrix that is used as uniquely identified Kronecker permutation matrix

\section{B. Pseudo Code}

Let us suppose two matrices $A$ and $B$ of $n x n$ dimensions. The generation of $B \otimes A$ from $A \otimes B$ is to formulated by using an $n^{2}$ permutation matrix. This permutation matrix can be formulated as follows:

1) Generate a natural number $n x n$ dimensional matrix having $n^{2}$ entities.

2) Reshape the matrix column wise in such a way that each $(n+1)$ st column lies beneath the nth column.

3) Make this column vector into a row vector.

4) The entities of this row vector point out towards the position of ' 1 ' at a specific row/column. Write ' 1 ' at every place pointing by the row vector and all other entries besides these locations are ' 0 's.

5) This generated matrix is the required uniquely identified permutation matrix.

The implementation of the pseudo code for $4 \times 4 \mathrm{PM}$ is as follows

$$
\begin{aligned}
& \stackrel{1^{s t}}{\rightarrow}\left[\begin{array}{ll}
1 & 2 \\
3 & 4
\end{array}\right] \stackrel{2^{n d}}{\rightarrow}\left[\begin{array}{l}
1 \\
3 \\
2 \\
4
\end{array}\right] \stackrel{3^{r d}}{\rightarrow}\left[\begin{array}{llll}
1 & 3 & 2 & 4
\end{array}\right] \\
& \stackrel{4^{t h}}{\rightarrow}\left[\begin{array}{llll}
1 & 0 & 0 & 0 \\
0 & 0 & 1 & 0 \\
0 & 1 & 0 & 0 \\
0 & 0 & 0 & 1
\end{array}\right] \stackrel{5^{t h}}{\rightarrow} \text { (Unique } P M \text { ) }
\end{aligned}
$$

Similarly for $16 \times 16 \mathrm{PM}$, it can be obtained by the swapping of natural number matrix rows/columns and following rows will come one after the other in $16 \times 16$ identity matrix as

$$
\begin{gathered}
{\left[1^{s t}, 5^{t h}, 9^{t h}, 13^{t h}, 2^{\text {nd }}, 6^{\text {th }}, 10^{\text {th }}, 14^{\text {th }}, 3^{r d}, 7^{\text {th }}, 11^{\text {th }},\right.} \\
\left.15^{t h}, 8^{t h}, 12^{\text {th }}, 16^{\text {th }}\right]
\end{gathered}
$$




\section{OBSERVATION AND RESUlTS}

In order to compare our proposed methodology we generated random matrices of order $4 \times 4$ till $20 \times 20$ for Kronecker Product, requiring permutation matrix of order 16 $\times 16$ up to $400 \times 400$ to calculate its reverse order. The Kronecker product for these matrices were calculated and afterwards derived from their reverse order product, by generating permutation matrix. It is observed the original KP and the one calculated using its reverse order using PM is always same and their difference gives the null matrix that verifies the applicability of this technique. For instance let us suppose two matrices of order $3 \times 3$.

$$
\mathrm{A}=\left[\begin{array}{lll}
a & b & c \\
d & e & f \\
g & h & i
\end{array}\right] \quad \& \mathrm{~B}=\left[\begin{array}{ccc}
j & k & l \\
m & n & o \\
p & q & r
\end{array}\right]
$$

$A \quad \mathrm{~A} \otimes B=\left[\begin{array}{lllllllll}a j & a k & a l & b j & b k & b l & c j & c k & c l \\ a m & a n & a o & b m & b n & b o & c m & c n & c o \\ a p & a q & a r & b p & b q & b r & c p & c q & c r \\ d j & d k & d l & e j & e k & e l & f j & f k & f l \\ d m & d n & d o & e m & e n & e o & f m & f n & f o \\ d p & d q & d r & e p & e q & e r & f p & f q & f r \\ g j & g k & g l & h j & h k & h l & i j & i k & i l \\ g m & g n & g o & h m & h n & h o & i m & i n & i o \\ g p & g q & g r & h p & h q & h r & i p & i q & i r\end{array}\right] \&$

$B \quad B \otimes A=\left[\begin{array}{ccccccccc}a j & b j & c j & a k & b k & c k & a l & b l & c l \\ d j & e j & f j & d k & e k & f k & d l & e l & f l \\ g j & h j & i j & g k & h k & i k & g l & h l & i l \\ a m & b m & c m & a n & b n & c n & a o & b o & c o \\ d m & e m & f m & d n & e n & f n & d o & e o & f o \\ g m & h m & i m & g n & h n & i n & g o & h o & i o \\ a p & b p & c p & a q & b q & c q & a r & b r & c r \\ d p & e p & f p & d q & e q & f q & d r & e r & f r \\ g p & h p & i p & g q & h q & i q & g r & h r & i r\end{array}\right]$

The Permutation Matrix of order $9 \times 9$ using the stated methodology will be:

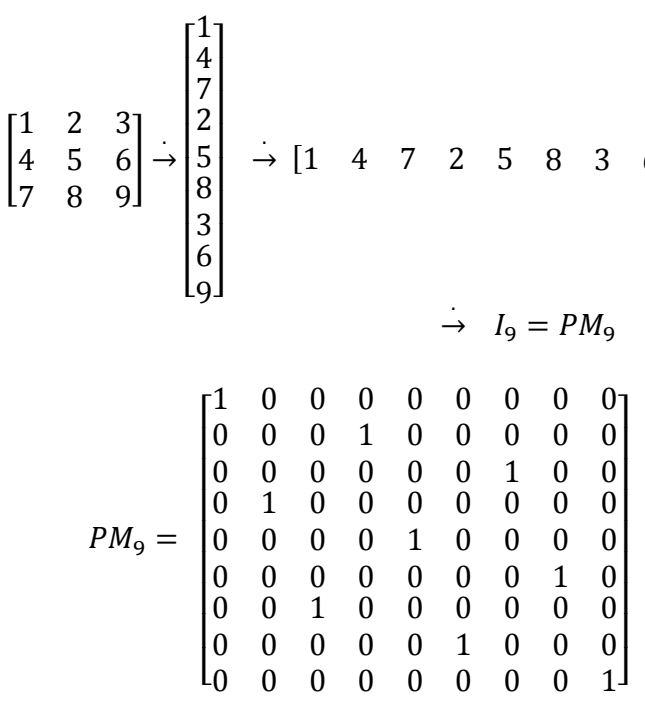

As stated in (8) \& (9),

$$
B \otimes A=P(A \otimes B) P^{T}
$$

where, $P=P^{-1}=P^{T}$

Substituting (9) in (8), it would become

$$
B \otimes A=P(A \otimes B) P
$$

So using the above equation,

$$
\begin{aligned}
& P\left[(\mathrm{~A} \otimes \mathrm{B}) P^{T}\right]= \\
& P M_{9}\left[\begin{array}{ccccccccc}
a j & a k & a l & b j & b k & b l & c j & c k & c l \\
a m & a n & a o & b m & b n & b o & c m & c n & c o \\
a p & a q & a r & b p & b q & b r & c p & c q & c r \\
d j & d k & d l & e j & e k & e l & f j & f k & f l \\
d m & d n & d o & e m & e n & e o & f m & f n & f o \\
d p & d q & d r & e p & e q & e r & f p & f q & f r \\
g j & g k & g l & h j & h k & h l & i j & i k & i l \\
g m & g n & g o & h m & h n & h o & i m & i n & i o \\
g p & g q & g r & h p & h q & h r & i p & i q & i r
\end{array}\right] \\
& =P M_{9}\left[\begin{array}{ccccccccc}
a j & b j & c j & a k & b k & c k & a l & b l & c l \\
a m & b m & c m & a n & b n & c n & a o & b o & c o \\
a p & b p & c p & a q & b q & c q & a r & b r & c r \\
d j & e j & f j & d k & e k & f k & d l & e l & f l \\
d m & e m & f m & d n & e n & f n & d o & e o & f o \\
d p & e p & f p & d q & e q & f q & d r & e r & f r \\
g j & h j & i j & g k & h k & i k & g l & h l & i l \\
g m & h m & i m & g n & h n & i n & g o & h o & i o \\
g p & h p & i p & g q & h q & i q & g r & h r & i r
\end{array}\right] \\
& =\left[\begin{array}{ccccccccc}
a j & b j & c j & a k & b k & c k & a l & b l & c l \\
d j & e j & f j & d k & e k & f k & d l & e l & f l \\
g j & h j & i j & g k & h k & i k & g l & h l & i l \\
a m & b m & c m & a n & b n & c n & a o & b o & c o \\
d m & e m & f m & d n & e n & f n & d o & e o & f o \\
g m & h m & i m & g n & h n & i n & g o & h o & i o \\
a p & b p & c p & a q & b q & c q & a r & b r & c r \\
d p & e p & f p & d q & e q & f q & d r & e r & f r \\
g p & h p & i p & g q & h q & i q & g r & h r & i r
\end{array}\right]=B \otimes A
\end{aligned}
$$

The results obtained are by calculating the reverse product directly, and by evaluating it through permutation matrix technique gives the same result, as the subtraction of the two, gives a null matrix, that intern proves the reliability of this methodology.

\section{CONCLUSION}

The paper gives a brief overview of Kronecker product and its implementation in finding the reverse order multiplication, as in formulation DFT in signal processing using a unique permutation matrix technique by finding it intuitively. This would lessen its computation cost by not calculating the reverse product being it to be computationally expensive algorithm and intern evaluating it by using the ground principles of matrix theory through a unique permutation matrix, from the same previously found Kronecker product. The computation cost to calculate the PM for reverse order $\mathrm{KP}$ will be minimum as the entities for an $n \times n$ unique PM can be depicted directly, without finding it from n! PM's.

\section{REFERENCES}

[1] A. K1lıçman and Z. Zhour, "On the connection between Kronecker and Hadamard convolution products of matrices and some applications," Journal of Inequalities and Applications, vol. 2009, no. 1, p. 736243 , 2009.

[2] G. Alexander, Kronecker Products and Matrix Calculus with Applications, New York: Halsted, 1981.

[3] F. Ding "Transformations between some special matrices," Computers \& Mathematics with Applications, vol. 59, no. 8, pp. 2676-2695, 2010.

[4] Z. A. A. Al-Zhour, "New techniques for solving some matrix and matrix differential equations," Ain Shams Engineering Journal, vol. 6 no. 1 , pp. 347-354, 2015

[5] A. V. Oppenheim, RW Schafer Digital Signal Processing, Englewood Cliffs, New Jersey: Prentice-Hall, vol. 6, 1975, pp. 125-136. 
[6] Y. Avilés, On Kronecker Products Signal Processing Algorithms and FPGA Computational Structures, 1999.

[7] M. Benzi and S. Valeria, "Approximation of functions of large matrices with Kronecker structure," Numerische Mathematik, vol. 135, no. 1, pp. 1-26, 2017.

[8] H. Evangelaras, C. Koukouvinos, and J. Seberry, "Applications of Hadamard matrices," Journal of Telecommunications and Information Technology, pp. 3-10, 2003.

[9] P. A. Regalia and K. S. Mitra, "Kronecker products, unitary matrices and signal processing applications," SIAM Review, vol. 31, no. 4, pp. 586-613, 1989

[10] A. Rövid, L. Szeid, I. Rudas, and P. Várlaki, "Image processing on tensor-product basis," Óbuda University e-Bulletin, vol. 2, no. 1, pp. 247-258, 2011.

[11] W. Steeb and H. Yorick, Matrix Calculus and Kronecker Product: A Practical Approach to Linear and Multilinear Algebra Second Edition, World Scientific Publishing Company, 2011.

[12] C. Jhurani, "Batched Kronecker product for 2-D matrices and 3-D arrays on NVIDIA GPUs," arXiv preprint arXiv:1304.7054, 2013.

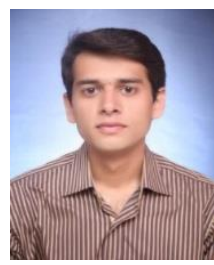

Muhammad G. Ali received his bachelor's in electrical computer engineering from COMSATS University in 2017. He has developed an on-board diagnostic tool for providing maintenance and security to vehicles in collaboration with Engr. Qasim B.

$\mathrm{He}$ has served in MEGAPLUS as a trainee engineer and is currently working as an asst. network/system administrator in a government organization Furthermore he is also pursuing his research in hybrid technologies for mathematical modeling for signal processing algorithms on hardware coded architectures.

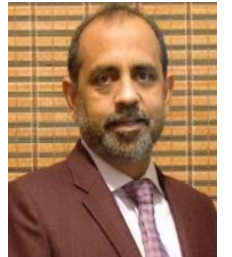

Shoab A. Khan received his bachelor's degree from College of Aeronautical Engineering, NED University in 1989 and the MS \& PhD in electrical and computer engineering from Georgia Institute of Tech, Atlanta, GA, USA in 1995. He is a professor and the head of Department of Computer \& Software Engineering Department in College of Electrical \& Mechanical Engineering, National University of Sciences \& Technology.

$\mathrm{He}$ is an inventor of 6 awarded US patents and has hundreds of international publications. His book on digital design is published by John Wiley \& Sons and is being followed in national and international universities. Dr. Shoab Ahmed Khan has more than 2 decades of industrial experience in companies in USA and Pakistan. He has been awarded Tamgh-e-Imtiaz (Civil), National Education Award 2001 and NCR National Excellence Award in Engineering Education.

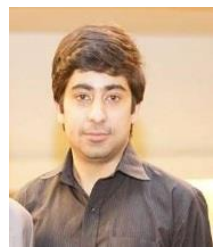

Sajid G. Khawaja received his bachelor's, master's degree in computer engineering and is a $\mathrm{PhD}$ holder from National University of Sciences and Technology, Pakistan.

$\mathrm{He}$ is currently serving as an assistant professor in National University of Sciences and Technology, College of Electrical and Mechanical Engineering. His area of interest is digital system designs and has many publications in his career. Furthermore he is also an inventor of 3 international/national patents. 